\title{
CLEO Results on Tau Michel Parameters
}

\author{
Alan J. Weinstein ${ }^{\mathrm{a}}$ \\ ${ }^{\text {a}}$ California Institute of Technology, Pasadena, CA 91125
}

We present measurements of the tau Michel Parameters made by the CLEO experiment. Three different analyses are performed: a spin-independent lepton spectrum analysis and a second spin-dependent analysis using $\ell^{ \pm}$vs. $\pi^{\mp} \pi^{0}$ events, and a third spin-dependent analysis using $\pi^{+}$vs. $\pi^{-}$events. the results are used to derive limits on the general four-fermion couplings, the mass of the charged Higgs in the MSSM, and a right-handed $W$ in left-right models. Many of these measurements are more precise than the PDG world averages.

\section{INTRODUCTION}

Heavy lepton $(\mu, \tau)$ decays are mediated (in the Standard Model) by the charged weak current, carried by $W^{ \pm}$force bosons with a $V-A$ Lorentz structure. As a consequence, such decays exhibit maximal parity violation, while conserving CP. The couplings of all the charged leptons to the $W^{ \pm}$are consistent with being exactly equal (universality).

Many extensions to the Standard Model predict small deviations from the $V-A$ law, and/or fermion universality. Such deviations can be observed in sensitive measurements of the Lorentz structure of the weak interaction. In leptonic decays of the $\mu$ and $\tau$ leptons, in which the outgoing neutrinos are unobserved, the dynamics of the decay can be described in terms of the Michel parameters [1]. The precise measurement of these parameters can be used to search for evidence of, or provide limits on, processes which go beyond the pure $V-A$ structure of the charged weak current. In particular, they are sensitive to the presence of small scalar currents (such as those mediated by the charged Higgs of the Minimal Supersymmetric extensions [2] to the Standard Model, MSSM), or small deviations from maximal parity violation (such as those mediated by the right-handed $W_{R}$ of left-right symmetric extensions [3] to the Standard Model).

In the decays of the $\tau$ to $\ell \nu \bar{\nu}$, information on the decay can be extracted from the shape of the momentum distribution of the lepton $\ell$, and from its angular distribution relative to the parent $\tau$ spin direction [1, 4. After integration over the unobserved neutrino momenta and the spin of $\ell$, and neglecting radiative effects we can write the charged lepton momentum spectrum as:

$$
\begin{aligned}
& \frac{1}{\Gamma} \frac{d \Gamma}{d x d \cos \theta}=\frac{x^{2}}{2} \times \\
& {\left[\left(12(1-x)+\frac{4 \rho}{3}(8 x-6)+24 \eta \frac{m_{\ell}}{m_{\tau}} \frac{(1-x)}{x}\right)\right.} \\
&\left.\quad \pm P_{\tau} \xi \cos \theta\left(4(1-x)+\frac{4}{3} \delta(8 x-6)\right)\right] \\
& \propto x^{2}\left[I(x \mid \rho, \eta) \pm P_{\tau} A(x, \theta \mid \xi, \delta)\right]
\end{aligned}
$$

where $\rho$ and $\eta$ are the spectral shape Michel parameters and $\xi$ and $\delta$ are the spin-dependent Michel parameters [1]; $x=E_{\ell} / E_{\max }$ is the daughter charged lepton energy scaled to the maximum energy $E_{\max }=\left(m_{\tau}^{2}+m_{\ell}^{2}\right) / 2 m_{\tau}$ in the $\tau$ rest frame; $\theta$ is the angle between the tau spin direction and the daughter charged lepton momentum in the $\tau$ rest frame; and $P_{\tau}$ is the polarization of the $\tau$. In the Standard Model (SM), the Michel Parameters have the values $\rho=3 / 4$, $\eta=0, \xi=1$ and $\delta=3 / 4$. Since $\tau^{+} \tau^{-}$events are produced with no net polarization at $e^{+} e^{-}$centerof-mass energies below the $Z^{0}$ mass, the lepton momentum spectrum alone is not sensitive to the spin-dependent parameters $\xi$ and $\delta$. These latter parameters are measurable by analyzing the decays of both taus in an event. In addition, semihadronic tau decays permit the measurement of the parameter $h_{\nu_{\tau}} \equiv \xi_{h}=-g_{v} g_{a} /\left(g_{v}^{2}+g_{a}^{2}\right)$, sometimes referred to as the "tau neutrino helicity", 
which takes the value +1 in the $V-A$ SM.

\section{CLEO DATA SAMPLE AND ANALY- SIS}

Here we report on measurements of these Michel parameters in three separate analyses performed on data from the CLEO II experiment [5] at Cornell's CESR collider, from the reaction $e^{+} e^{-} \rightarrow \tau^{+} \tau^{-}$at center of mass energy near 10.58 $\mathrm{GeV}$.

In the first analysis, events of the type $\ell^{-} \bar{\nu} \nu_{\tau}$ vs. $\pi^{+} \pi^{0} \bar{\nu}_{\tau}$ are selected" The $\pi^{ \pm} \pi^{0}$ system is used as a tag (to identify a tau pair event), and to estimate the tau direction in the lab. The lepton energy spectrum is measured and the parameters $\rho$ and $\eta$ are extracted for $\tau \rightarrow e \nu \nu$ and $\tau \rightarrow \mu \nu \nu$.

In the second analysis, the same $\ell^{-} \bar{\nu} \nu_{\tau} v s$. $\pi^{+} \pi^{0} \bar{\nu}_{\tau}$ sample used in the first analysis is again used. Here, the $\pi^{ \pm} \pi^{0}$ system is used to analyze the spin of the decaying $\tau^{ \pm}$. The spin correlations produce a correlation between the $\pi^{ \pm} \pi^{0}$ system from one tau and the momentum of the charged lepton from the other tau. A fitter which extracts all the available information from the full measured kinematics of these events is used to measure the parameters $\rho, \quad \xi, \quad \delta$, and $\left|h_{\nu_{\tau}}\right|$. The value of $\rho$ obtained in this analysis supersedes that of the first, and no attempt is made to re-measure $\eta$.

In the third analysis, events of the type $\pi^{-} \nu_{\tau}$ vs. $\pi^{+} \bar{\nu}_{\tau}$ are selected. The tau spin correlations produce correlations in the momenta of the two pions, which are used to extract $\left|h_{\nu_{\tau}}\right|^{2}$.

The first and second analyses use $3.0 \times 10^{6}$ produced tau pairs, while the third uses only $1.5 \times 10^{6}$.

For the first two analyses, we select events where one $\tau$ decays leptonically $\left(e^{\mp}\right.$ or $\left.\mu^{\mp}\right)$, and the other decays to $\pi^{ \pm} \pi^{0} \nu_{\tau}$. The $\pi^{ \pm} \pi^{0} \nu_{\tau}$ mode is used because it has a large branching fraction, negligible background from $e^{+} e^{-} \rightarrow e^{+} e^{-}, \mu^{+} \mu^{-}$, or $q \bar{q}$, and a high, well-understood trigger efficiency.

${ }^{1}$ Throughout this paper, charge conjugate processes are implied.

\section{LEPTON SPECTRUM ANALYSIS}

In the first analysis [6], the $\rho^{\mp} \rightarrow \pi^{\mp} \pi^{0}$ decay is used to estimate the $\tau^{\mp}$ flight direction. The angle between the $\rho^{\mp}$ momentum and the tau momentum in the lab can be calculated (in the absence of radiative effects). In approximately $60 \%$ of the events, this angle is less than $11^{\circ}$ and is used to estimate the $\tau^{\mp}$ direction, and thus the recoiling $\tau^{ \pm}$direction in the lab. This permits us to boost the daughter charged lepton $\ell^{ \pm}$momentum into the tau rest frame so estimated (the "pseudo-rest frame" (7]). For events in which the above angle is greater than $11^{\circ}$, the $\ell^{ \pm}$momentum is analyzed in the lab frame.

Muons with lab frame momentum $p_{\mu}<1.5$ $\mathrm{GeV} / \mathrm{c}$ are not well identified in the CLEO muon detectors. We can select decays with one charged track which is inconsistent with being an electron, and which has no extra photons from $\pi^{0}$ decays. Such decays are most likely either $\tau \rightarrow \mu \nu \nu$ or $\tau \rightarrow \pi \nu$. If the energy of the charged track in the pseudo-rest frame $E_{\mu}<0.6 m_{\tau}$ then the event is kinematically inconsistent with being a $\tau \rightarrow \pi \nu$ decay. We thus obtain a sample of $\tau \rightarrow \mu \nu \nu$ decays with low momentum muons (particularly sensitive to the $\eta$ parameter), with an estimated purity of $96 \%$.

The final $\tau \rightarrow e \nu \nu$ decay sample consists of 18587 events analyzed in the pseudo-rest frame and 12981 analyzed in the lab frame. The final $\tau \rightarrow \mu \nu \nu$ decay sample consists of 12580 events with muons identified in the CLEO muon detector and 2931 events with muons identified kinematically and analyzed in the pseudo-rest frame, and 9186 events with muons identified in the CLEO muon detector and analyzed in the lab frame. Backgrounds from pions misidentified as hadrons are measured from the data and subtracted. All other backgrounds are estimated by the Monte Carlo to be negligible.

The resulting momentum spectra were fitted to Monte Carlo [8] distributions, including all radiative effects, $\rho^{\mp}$ dynamics, spin correlations, and detector efficiency and resolution. The two electron spectra were fitted with one parameter, $\rho_{e}$, with the result $\rho_{e}=0.732 \pm 0.015$. The three muon spectra were fitted with two parameters, 
$\rho_{\mu}$ and $\eta_{\mu}$, with the results $\rho_{\mu}=0.747 \pm 0.055$ and $\eta_{\mu}=0.010 \pm 0.174$; these values are strongly correlated (see Fig. 3). All five spectra were fitted simultaneously, with the constraint that $\rho_{e}=\rho_{\mu} \equiv \rho_{e \mu}$, with the two parameters $\rho_{e \mu}$ and $\eta_{e \mu}$. Note, here, that $\eta_{e \mu}$ is the value of $\eta$ appropriate for the muon decays, since the electron decays are largely insensitive to the value of $\eta$. The subscripted $\eta_{e \mu}$ is simply a reminder that the result is obtained with the $\rho_{e}=\rho_{\mu}$ constraint. The results are: $\rho_{e \mu}=0.735 \pm 0.014$ and $\eta_{e \mu}=-0.015 \pm 0.066$.

The spectra in the pseudo-rest frame, and the fit results, are shown in Figs. 1 and 2. the results are summarized in Fig. 3. Many systematic studies and fit variations were performed, all consistent with each other. The results quoted above include systematic errors. All these results are consistent with the Standard Model expectation that $\rho_{e}=\rho_{\mu}=3 / 4$ and $\eta_{\mu}=0$.

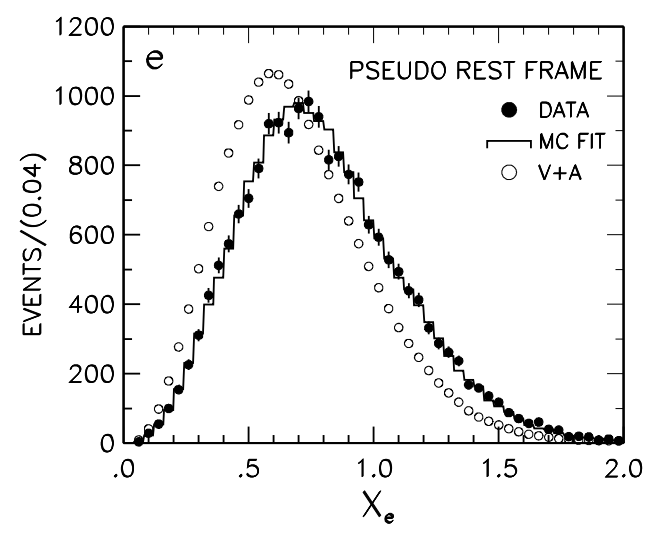

Figure 1. The electron scaled pseudo rest frame energy spectra (solid points are data, histogram is the fit function). Open circles give the $\mathrm{MC}$ predicted spectrum for $V+A$. Events with $X>1$ result from the imperfect reconstruction of the $\tau$ direction.

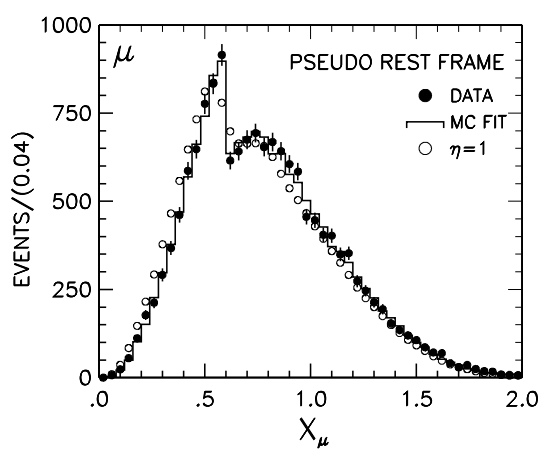

Figure 2. The muon scaled pseudo rest frame energy spectra (solid points are data, histogram is the fit function). Open circles give the MC predicted spectrum for $\eta=1$. The addition of the low momentum muons results in the discontinuity observed at $X_{\mu}=0.6$.

\section{SPIN-DEPENDENT ANALYSIS}

The second analysis [9] uses the same event sample as in the first, $\ell^{-} \bar{\nu} \nu_{\tau}$ vs. $\pi^{+} \pi^{0} \bar{\nu}_{\tau}$, but the kinematically-identified low-momentum muons are not used. We analyze the $\tau^{+} \rightarrow$ $\pi^{+} \pi^{0} \bar{\nu}_{\tau}$ decay to extract information on the $\tau^{+}$ spin orientation; the QED-predicted spin correlations in $e^{+} e^{-} \rightarrow \tau^{+} \tau^{-}$thus give information on the recoil $\tau^{-}$spin direction. We then analyze the momentum of the charged lepton in the $\tau^{-} \rightarrow \ell^{-} \bar{\nu}_{\ell} \nu-\tau$ decay in order to extract $\xi$ and $\delta$, separately for electronic and muonic decays. At the same time, we measure the magnitude of the tau neutrino helicity $h_{\nu_{\tau}}$, and re-measure the $\rho$ parameter. We assume the value of $\eta$ as measured from the first analysis. We also select 11177 events of the type $\pi^{-} \pi^{0} \nu_{\tau}$ vs. $\pi^{+} \pi^{0} \bar{\nu}_{\tau}$, to extract $\left|h_{\nu_{\tau}}\right|$ with greater precision.

The $\rho^{+} \rightarrow \pi^{+} \pi^{0}$ decay is used to analyze the parent tau spin orientation. The energy of the $\pi^{+} \pi^{0}$ system, and the angle of the pion momentum in the $\pi^{+} \pi^{0}$ rest frame relative to the $\pi^{+} \pi^{0}$ momentum in the lab frame can be combined to give a "polarimeter" variable [10] $\omega=$ $\left\langle\vec{p}_{\tau} \cdot \vec{H}_{\tau}\right\rangle_{\phi_{\nu_{\tau}}}=\omega\left(E_{\rho}, \cos \theta(\rho \rightarrow \pi \pi)\right)$. Clear cor- 


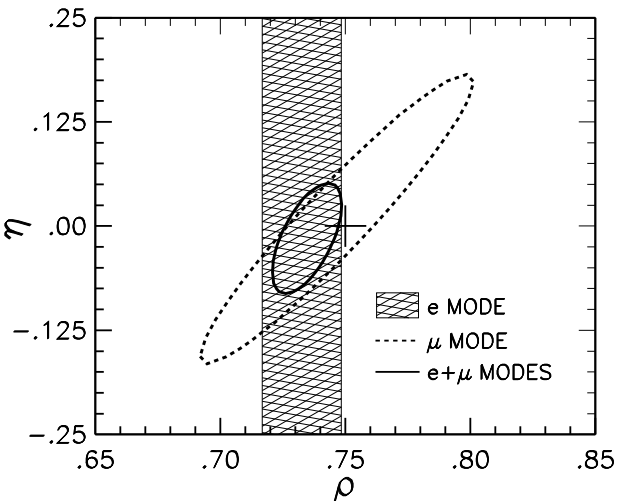

Figure 3. Results from the lepton spectrum analysis. The shaded band denotes the electron mode result, the large dotted contour indicates the muon mode $1 \sigma$ error ellipse obtained in the $\eta-\rho$ plane, and the small dark ellipse indicates the corresponding $1 \sigma$ result obtained from the simultaneous fit to both modes. The Standard Model expectation is indicated by the cross.

relations between the value of $\omega$ from the $\tau^{+}$decay and the charged lepton momentum from the $\tau^{-}$decay are observed, consistent with Standard Model expectations.

To make full use of all the available kinematical information, we do a multi-dimensional likelihood fit to all of the observed three-momenta in the lab, using the full production and decay matrix element, including the effects of radiation (ISR/FSR, decay, and external bremsstrahlung). Schematically, for the $\mu-v s . \rho$ sample,

$$
\begin{aligned}
|\mathcal{M}|^{2}= & H_{1} \times P \times\left[L_{1}+\rho L_{2}+\eta L_{3}\right] \\
& +h_{\nu_{\tau}} H_{1 \alpha}^{\prime} \times C^{\alpha \beta} \times\left[\xi L_{1 \beta}^{\prime}+\xi \delta L_{2 \beta}^{\prime}\right] .
\end{aligned}
$$

Here, $H_{1}$ describes the spin-independent part of the $\tau \rightarrow \pi \pi^{0} \nu$ decay rate, $L_{1}$ through $L_{3}$ describe the spin-independent part of the $\tau \rightarrow \ell \nu \nu$ decay rate (with explicit Michel parameter dependence), and $P$ describes the spin-independent part of the $\tau^{+} \tau^{-}$production. $H_{1 \alpha}^{\prime}, L_{1 \beta}^{\prime}$ and $L_{2 \beta}^{\prime}$, and $C^{\alpha \beta}$ describe the analogous spin-dependent quantities. All are functions of the momenta of all the observed particles.

Evaluation of the likelihood per event requires integration over the unobserved neutrinos and radiated photons: a 22 dimensional integral. This is performed using a unique "reverse Monte Carlo" technique. All major "backgrounds" $\left(\pi, K \pi, \pi 2 \pi^{0} \rightarrow \pi \pi^{0}, \pi \rightarrow \mu\right)$ are modeled, with full Michel parameter dependence. The detector efficiency and resolution is fully modeled. The fit procedure was tested with many ensembles of Monte Carlo events with all three topologies $(e-v s . \rho, \mu-v s . \rho, \rho-v s .-\rho)$, including backgrounds; no bias was observed.

A measurement of the spin-dependent Michel parameters allows one to distinguish the Standard Model $V-A$ interaction (left-handed $\nu_{\tau}$ ) from $V+A$ (right-handed $\nu_{\tau}$ ). The probability that a right-handed (massless) tau neutrino participates in the decay can be expressed as

$$
P_{R}^{\tau}=1 / 2[1+1 / 9(3 \xi-16 \xi \delta)],
$$

and $P_{R}^{\tau}=0$ for the SM $V-A$ interaction.

The results of the fits to the $e-v s . \rho$ sample are:

$$
\begin{array}{rll}
\rho_{e}=0.747 \pm 0.012 \pm 0.004 & & (S M=3 / 4) \\
\xi_{e}=0.979 \pm 0.048 \pm 0.016 & & (S M=1) \\
\xi_{e} \delta_{e}=0.720 \pm 0.032 \pm 0.010 & & (S M=3 / 4) .
\end{array}
$$

All measures of goodness-of-fit yield satisfactory results. From the distribution of the likelihoods for each event as compared to Monte Carlo expectations, the confidence level (CL) for the fit is $73 \%$. Comparisons of various kinematical quantities between the data and the results of the fit are shown in Fig. 1 . We extract the limit $P_{\tau}^{R}<0.065$ at $90 \%$ CL. The last three plots in this figure clearly demonstrate the spin correlations and the effectiveness of the fit in modeling them.

For the $\mu-v s . \rho$ sample, the results (using $\eta_{\mu}=$ 0.010 from the first analysis) are:

$$
\begin{aligned}
\rho_{\mu} & =0.750 \pm 0.017 \pm 0.045 \\
\xi_{\mu} & =1.054 \pm 0.069 \pm 0.047 \\
\xi_{\mu} \delta_{\mu} & =0.786 \pm 0.041 \pm 0.032
\end{aligned}
$$

with a confidence level for the fit of $21 \%$. We extract the limit $P_{\tau}^{R}<0.067$ at $90 \%$ CL. 

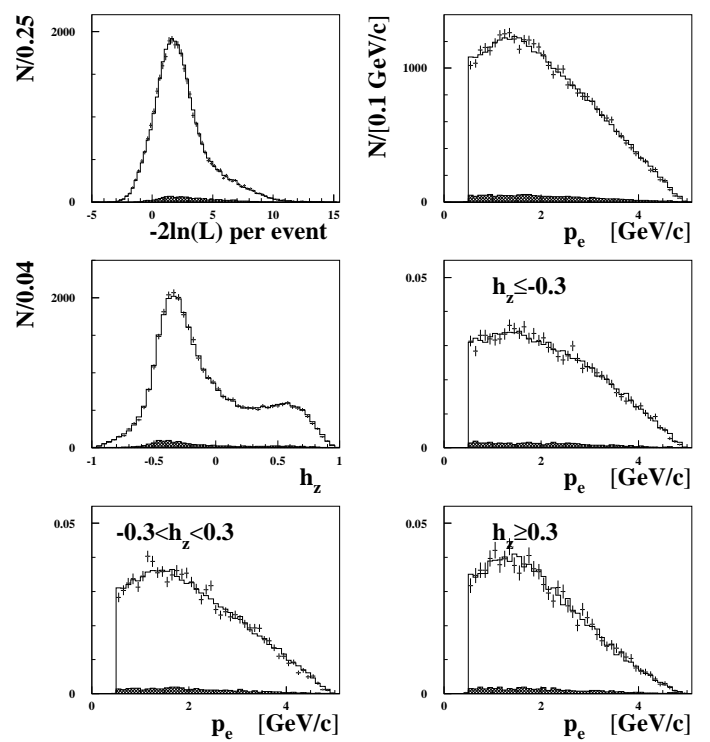

Figure 4. Comparison of the $e-v s . \rho$ data (points) with the fit results (histograms) for the following quantities: the $-2 \ln \mathcal{L}$ (log-likelihood) per event; the electron momentum in the lab; the polarimeter variable $\omega \equiv h_{Z}$ for the $\rho$ decay, and the electron momentum for three ranges of polarimeter variable of the recoiling $\rho$ decay: $\omega \leq-0.3$, $-0.3<\omega<0.3$, and $\omega \geq 0.3$.

For the $\rho-v s . \rho$ sample, the results are:

$$
h_{\nu_{\tau}}^{2}=0.995 \pm 0.010 \pm 0.003 \quad(S M=1)
$$

with a confidence level for the fit of $9 \%$.

The following sources of systematic errors were considered: Monte Carlo statistics; lepton identification efficiency; spin analyzer efficiency; modeled backgrounds; remaining unmodeled backgrounds; contribution of $\rho^{\prime}$ to the $\pi \pi^{0}$ dynamics; the value of the Michel parameter $\eta$; detector energy, momentum, and angular resolution; and modeled and unmodeled radiation effects. There is no sign of significant systematic bias, and Monte Carlo statistics remains the largest source of systematic error in most cases.

Under the assumption of lepton universality, we can constrain $\rho_{e}=\rho_{\mu} \equiv \rho_{e \mu}$, and similarly for $\xi$ and $\xi \delta$. We then obtain:

$$
\begin{aligned}
\rho_{e \mu} & =0.747 \pm 0.010 \pm 0.006 \\
\xi_{e \mu} & =1.007 \pm 0.040 \pm 0.015 \\
(\xi \delta)_{e \mu} & =0.745 \pm 0.026 \pm 0.009 .
\end{aligned}
$$

We extract the limit $P_{\tau}^{R}<0.044$ at $90 \%$ CL.

These results are consistent with the Standard Model Prediction, and are, in most cases, more precise than the world average values 11]. These results supersede those on $\rho_{e}, \rho_{\mu}, \rho_{e \mu}$ from the first analysis.

\section{SPIN CORRELATIONS IN $\pi^{+}$vs. $\pi^{-}$}

In the third analysis [12], we again exploit the fact that in $e^{+} e^{-} \rightarrow \gamma^{*} \rightarrow \tau^{+} \tau^{-}$at $10 \mathrm{GeV}$, the $\tau$ spins are $95 \%$ anti-correlated. If both taus decay semi-hadronically, then in the lab frame, the differential distribution of the energies of the two hadronic systems scaled to the beam energy, $x_{ \pm} \equiv E_{ \pm} / E_{b}$ is given by:

$$
\frac{d^{2} \sigma}{d x_{+} d x_{-}}=F_{1}\left(x_{+}, x_{-}\right)+\left|h_{\nu_{\tau}}\right|^{2} F_{2}\left(x_{+}, x_{-}\right)
$$

where the functions $F_{1}$ and $F_{2}$ are well known and are particularly simple for $\pi^{+}$vs. $\pi^{-}$events.

We select events of the type $\left(\tau^{-} \rightarrow \pi^{-} \nu_{\tau}\right) v s$. $\left(\tau^{+} \rightarrow \pi^{+} \bar{\nu}_{\tau}\right)$, with no extra photons. In order to suppress $\mu \rightarrow \pi$ backgrounds, we require the pions to shower in the calorimeter. We use our limited $\mathrm{dE} / \mathrm{dx}$ separation to suppress $\tau \rightarrow K^{ \pm} \nu$. Kinematical cuts suppress two-photon events and other backgrounds. From $1.5 \times 10^{6}$ produced tau pairs, we obtain $2041 \pi^{+}$vs. $\pi^{-}$candidates. The distribution of scaled energies $x_{+} v s . x_{-}$is given in Fig. 5 .

From a fit to this distribution (and related ones), we obtain $\left|h_{\nu_{\tau}}\right|=1.03 \pm 0.06 \pm 0.04$, consistent with the SM expectation of 1 .

\section{DISCUSSION OF RESULTS}

From these results, limits can be obtained on the complex couplings in the generalized fourfermion interaction model [1, 位; these are shown in Fig. 6. the couplings are shown normalized to their maximum value (hence the prime'), and 


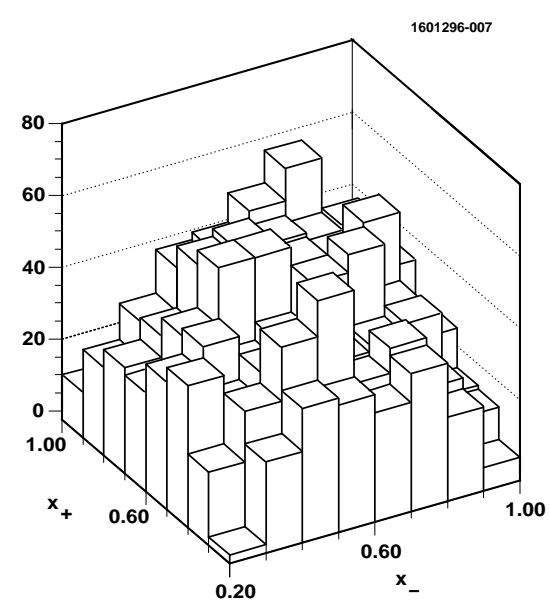

Figure 5. The raw correlation between the scaled energies $x_{ \pm} \equiv E_{ \pm} / E_{b}$ of the two pions in $\left(\tau^{-} \rightarrow\right.$ $\left.\pi^{-} \nu_{\tau}\right)$ vs. $\left(\tau^{+} \rightarrow \pi^{+} \bar{\nu}_{\tau}\right)$ events.

$g_{L L}^{V \prime}$ is fixed to be real. The new results improve the limits on couplings to right-handed tau neutrinos, but are unable to distinguish amongst the couplings to left-handed neutrinos without independent information such as a measurement of the cross-section $\sigma\left(\nu_{\tau} e \rightarrow \tau \nu_{e}\right)$. In the $V-A$ Standard Model, all of the couplings are zero except $g_{L L}^{V \prime}=1$. The results are clearly consistent with the Standard Model, but are still at the level of precision achieved for muon decays.

\subsection{Limits on Charged Higgs}

In the minimal supersymmetric extension to the Standard Model (MSSM), a charged Higgs boson will contribute to the decay of the tau, interfering with the left-handed $W^{-}$diagram, and producing a non-zero value for $\eta$. For $\tau \rightarrow \mu \nu \nu$,

$$
\eta=-\frac{m_{\tau} m_{\mu} \tan ^{2} \beta}{2 m_{H}^{2}},
$$

and similar formulas exist for $\xi$ and $\xi \delta$. With the measurements reported here of $\eta, \xi$, and $\xi \delta$ combined, we can extract the limit $m_{H^{ \pm}}>$ $1.02 \tan \beta \mathrm{GeV} / \mathrm{c}^{2}$ at $90 \% \mathrm{CL}$. This limit is significant in comparison with direct search limits, in the region $\tan \beta \gtrsim 200[13]$.

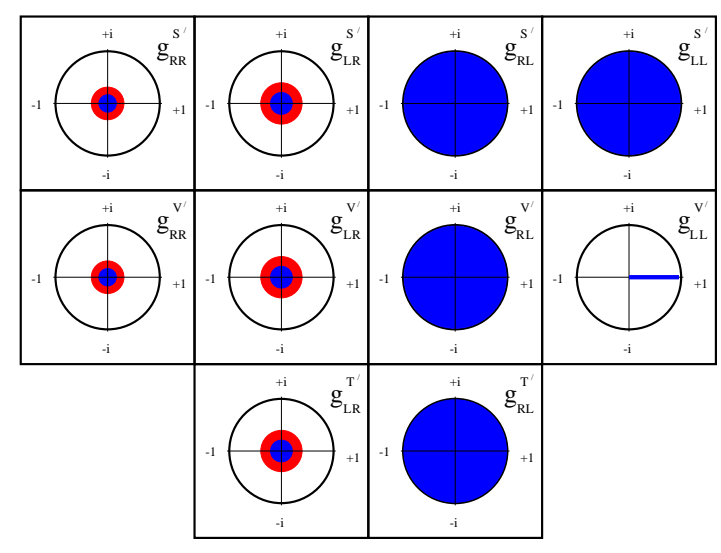

Figure $6 . \quad 90 \%$ confidence limits on the reduced coupling constants $g_{\epsilon \mu}^{\gamma^{\prime}}=g_{\epsilon \mu}^{\gamma} / \max \left(g_{\epsilon \mu}^{\gamma}\right)$, in the complex plane. The circles with unit radii indicate the maximum possible values, the other solid circles indicate the constraints obtained using present world average results, and the hatched regions indicate the new constraints obtained after including the results presented here.

\section{2. $W_{R}$ in Left-right symmetric models}

In left-right symmetric models [3], there are two sets of weak charged bosons $W_{1}^{ \pm}$and $W_{2}^{ \pm}$, which mix to form the observed "light" lefthanded $W_{L}^{ \pm}$and a heavier (so far, unobserved) right-handed $W_{R}^{ \pm}$. The parameters in these models are $\alpha=M\left(W_{1}\right) / M\left(W_{2}\right)$ (=0 in the $\mathrm{SM}$ ), and $\zeta=$ mixing angle, $=0$ in the SM. The heavy right-handed $W_{R}^{ \pm}$will contribute to the decay of the tau, interfering with the left-handed $W^{-}$diagram, and producing deviations from the Standard Model values for the Michel parameters $\rho$ and $\xi$.

By using the results of these analyses assuming lepton universality, especially the limit on $P_{R}^{\tau}$ (the probability that a right-handed tau neutrino participates in the decay), we can obtain limits on $\alpha$ and $\beta$ in these models, shown in Fig. 7. For mixing angle $\zeta=0$, we obtain $m_{R}>304 \mathrm{GeV} / \mathrm{c}^{2}$ at $90 \% \mathrm{CL}$, and for free mixing angle $\zeta$, we obtain $m_{2}>260 \mathrm{GeV} / \mathrm{c}^{2}$ at $90 \% \mathrm{CL}$. 


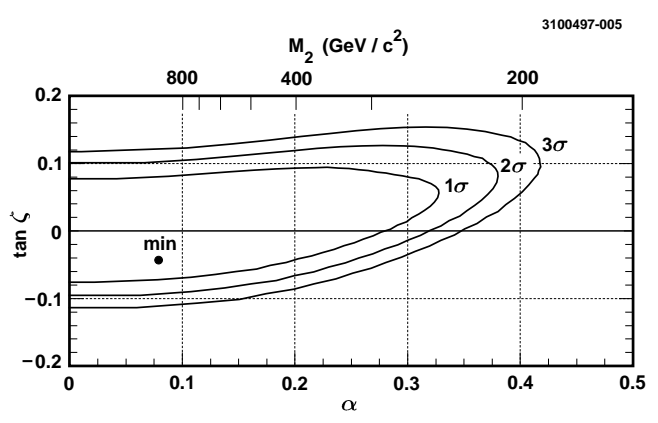

Figure 7. Limits on the mass ratio $\alpha$ and the mixing angle $\zeta$ of a left-right symmetric model.

\section{SUMMARY}

Using data from the reaction $e^{+} e^{-} \rightarrow \tau^{+} \tau^{-}$at center of mass energy near $10.58 \mathrm{GeV}$ collected with the CLEO II detector, we have studied the Lorentz structure of the $\tau-W-\nu_{\tau}$ couplings. we use three different analyses to extract measurements of the Michel Parameters $\rho, \eta, \xi, \delta$, and the tau neutrino helicity $h_{\nu_{\tau}}$. All of the measurements are consistent with the predictions from the pure $V-A$ Standard Model, at the $\sim$ few $\%$ level. They are, in most cases, more precise than the previous world averages.

From these measurements, we extract limits on the non-Standard Model complex couplings in the generalized four-fermion interaction model. We set a limit on the mass of the charged Higgs in MSSM models, significant for large $\tan \beta$. We set limits on the mass and mixing angle of a heavy right-handed $W$ predicted in left-right symmetric models.

In addition, we also present at this conference a precision measurement of the parity violating sign of $h_{\nu_{\tau}}$ in $\tau \rightarrow 3 \pi \nu_{\tau}$ decay [14].

Our results are still statistics limited, and CLEO II already has 3 times more data collected and now available for analysis. It is worth our while to continue improving the precision of these measurements, to search for small effects due to high energy scale physics.

\section{ACKNOWLEDGEMENTS}

We gratefully acknowledge the effort of the CESR staff in providing us with excellent luminosity and running conditions. This work was supported by the U.S. NSF and DoE. I thank the conference organizers for a thoroughly stimulating and pleasant conference.

\section{REFERENCES}

1. L. Michel, Proc. Phys. Soc. London A 63514 (1950); T. Kinoshita and A. Sirlin, Phys. Rev. 108844 (1957); C. Bouchiat and L. Michel, Phys. Rev. 106170 (1957); L. Okun and A. Rudik, J. Expt. Theor. Phys. (USSR) 32627 (1957) [Sov. Phys. JETP 6520 (1957)].

2. H. E. Haber, G. L. Kane and T. Sterling, Nucl. Phys. B 161493 (1979); J. F. Gunion and H. E. Haber, Nucl. Phys. B 2721 (1986); A. Stahl, Phys. Lett. B 324 (1994) 121-124.

3. M. A. B. Bég, R. V. Budny, R. Mohapatra and A. Sirlin, Phys. Rev. Lett. 381252 (1977).

4. F. Scheck, Phys. Rep. 44 (187 1978); W. Fetscher, Phys. Rev. D42 1544 (1990).

5. CLEO Collaboration, Y. Kubota et al., Nucl. Inst. Meth. A320 66 (1992).

6. CLEO Collaboration, R. Ammar, et al., Phys.Rev.Lett 78, 4686 (1997).

7. ARGUS Collab, H. Albrecht et al., Phys. Lett. B341 441 (1995).

8. KORALB v 2.2: S. Jadach and Z. Was, Comput. Phys. Commun. 36 (1985) 191. TAUOLA v2.4: S. Jadach, J. H. Kuhn and Z. Was, CERN-TH-5856/90 (1990). PHOTOS v1.06: E. Barberio, B. van Eijk and Z. Was, CERN-TH-5857/90 (1990).

9. CLEO Collaboration, J. Alexander, et al., Phys.Rev D56, 5320 (1997).

10. M. Davier, L. Duflot, F. Le Diberder and A. Rougé, Phys. Lett. B306 411 (1993).

11. Particle Data Group, R.M. Barnett et al., Phys. Rev. D54, 1 (1996).

12. CLEO Collaboration, T. Coan, et al., Phys.Rev. D55, 7291 (1997).

13. A. Stahl, Phys. Lett. B 324121 (1994).

14. M. Schmidtler, these proceedings. 